\title{
Cultural Adaptation of Scalable Psychological Interventions: A New Conceptual Framework
}

\author{
Eva Heim ${ }^{\mathrm{a}}$, Brandon A. Kohrt ${ }^{\mathrm{b}}$ \\ [a] Department of Psychology, University of Zurich, Zurich, Switzerland. [b] Department of Psychiatry, George \\ Washington University, Washington, DC, USA.
}

Clinical Psychology in Europe, 2019, Vol. 1(4), Article e37679, https://doi.org/10.32872/cpe.v1i4.37679

Received: 2019-06-25 • Accepted: 2019-11-01 • Published (VoR): 2019-12-17

Handling Editor: Cornelia Weise, Philipps-University of Marburg, Marburg, Germany

Corresponding Author: Eva Heim, University of Zurich, Department of Psychology, Binzmuehlestrasse 14/17, 8050 Zurich, Switzerland. E-mail: e.heim@psychologie.uzh.ch

\begin{abstract}
Background: The worldwide mental health treatment gap calls for scaling-up psychological interventions, which requires effective implementation in diverse cultural settings. Evidence from the field of global mental health and cultural clinical psychology indicates cultural variation in how symptoms of common mental disorders are expressed, and how culturally diverse groups explain the emergence of such symptoms. An increasing number of studies have examined to what extent cultural adaptation enhances the acceptability and effectiveness of psychological interventions among culturally diverse groups. To date, this evidence is inconclusive, and there is a lack of studies that dismantle the multiple types of modifications involved in cultural adaptation.

Method: Based on empirical evidence from ethnopsychological studies, cultural adaptation research, and psychotherapy research, the present paper offers a new conceptual framework for cultural adaptation that lays the groundwork for future empirical research.

Results: The cultural adaptation framework encompasses three elements: i) cultural concepts of distress; ii) treatment components; and iii) treatment delivery. These three elements have been discussed in literature but rarely tested in methodologically rigorous studies. Innovative research designs are needed to empirically test the relevance of these adaptation elements, to better understand the substantial modifications that enhance acceptability and effectiveness of psychological interventions.

Conclusion: Using a theory-driven approach and innovative experimental designs, research on cultural adaptation has the potential not only to make psychological treatments more accessible for culturally adverse groups, but also to further advance empirical research on the basic question about the "key ingredients" of psychotherapy.
\end{abstract}

\section{Keywords}

cultural adaptation, psychological interventions, culturally diverse groups, migrant populations 


\section{Highlights}

- The phenomenology of common mental disorders, as well as mind-body concepts, vary across cultures.

- Cultural adaptation may enhance the acceptability and effectiveness of psychological interventions.

- There is a lack of empirical evidence on the substantial modifications in cultural adaptation.

- Theory-driven, experimental approaches are needed in cultural adaptation research.

On 10 October 2017, Mental Health Europe celebrated World Mental Health Day in the European Parliament. Participants in this conference discussed the urgent need to support the mental health of refugees, migrants and asylum seekers (Mental Health Europe, 2017). According to United Nations, 180'000 migrants arrived in Europe in 2017, and 134'000 in 2018 (UN Dispatch, 2018). Prevalence rates of common mental disorders such as depression, anxiety, and post-traumatic stress disorder (PTSD) are high among immigrants in Europe, and particularly among survivors of armed conflicts (Bogic, Njoku, \& Priebe, 2015; Priebe, Giacco, \& El-Nagib, 2016).

Worldwide, there is a large mental health treatment gap, i.e. a high number of people in need of treatment who have not received adequate treatment. The treatment gap for common mental disorders is around $60 \%$ in high-income countries, $65 \%$ in upper-middle income countries, and over $80 \%$ in lower-middle income countries (Alonso et al., 2018; Thornicroft et al., 2017). Although the treatment gap is lower in high-income countries, there are specific barriers to mental health care for culturally diverse groups, which include poor command of the host country language, cultural beliefs about mental health, lack of trust in mental health services, and mental health related stigma (Priebe et al., 2016). The Lancet Commission on Global Mental Health and Sustainable Development (Patel et al., 2018) calls for action to scale up mental health services as an essential component of universal health coverage.

In response to the worldwide treatment gap, WHO and other research groups have invested in developing a series of potentially scalable psychological interventions (WHO, 2017). Scalability is defined as "The ability of a health intervention shown to be efficacious on a small scale and or under controlled conditions to be expanded under real world conditions to reach a greater proportion of the eligible population, while retaining effectiveness" (Milat, King, Bauman, \& Redman, 2013, p. 289).

One particular question for scaling-up concerns the extent to which results from one cultural group can be transferred to another. Ethnic minorities are generally underrepresented in clinical trials in high-income countries (Hussain-Gambles, Atkin, \& Leese, 2004; La Roche \& Christopher, 2008; Wendler et al., 2005). There is an ongoing debate in litera- 
ture on the extent to which psychological interventions developed in Western, Educated, Industrialized, Rich, and Democratic (WEIRD) societies (Henrich, Heine, \& Norenzayan, 2010) require cultural adaptation to be effective for the treatment of common mental disorders among culturally diverse groups. Literature indicates cultural variety in how symptoms of common mental disorders are expressed (Haroz et al., 2017; Kohrt et al., 2014), and how different cultural groups explain the emergence of such symptoms, thereby revealing their (implicit) assumptions about mind-body relationships, and religious or spiritual beliefs (e.g., Kohrt \& Hruschka, 2010).

Despite such cultural variance in symptoms and assumed causes, meta-analytic evidence suggests that evidence-based psychological interventions are effective for the treatment of common mental disorders among culturally diverse groups (Cuijpers, Karyotaki, Reijnders, Purgato, \& Barbui, 2018; Singla et al., 2017). But to what extent cultural adaptation can further enhance the acceptability and effectiveness of such interventions is subject to current debate in literature.

\section{Cultural Adaptation of Psychological Interventions}

Bernal, Jiménez-Chafey, and Domenech Rodríguez (2009) define cultural adaptation as "the systematic modification of an evidence-based treatment (EBT) or intervention protocol to consider language, culture, and context in such a way, that it is compatible with the client's cultural patterns, meanings, and values" (p. 362). Cultural adaptation can range from relatively low investment of resources (e.g., adaptation of illustrations or case examples) to adaptations which require a large amount of time and human resources, e.g. adaptation to cultural concepts of distress (Kohrt et al., 2014).

Bernal and colleagues (Bernal, Bonilla, \& Bellido, 1995; Bernal \& Sáez-Santiago, 2006) developed a framework for cultural adaptation of psychological interventions which encompasses eight elements: (a) language, (b) therapeutic relationship, (c) metaphors, (d) content of intervention, (e) concept of illness, (f) treatment goals, (g) delivering methods, and $(\mathrm{h})$ context. Meta-analytic evidence suggests that culturally adapted psychological interventions are effective when compared to a variety of control conditions $(d=0.45)$ (Griner \& Smith, 2006), and more effective than unadapted versions of the same intervention in direct comparison $(g=.52)$ (Hall, Ibaraki, Huang, Marti, \& Stice, 2016). Moreover, two meta-analyses showed that effect sizes increased with the number of implemented adaptation elements according to the Bernal framework (Harper Shehadeh, Heim, Chowdhary, Maercker, \& Albanese, 2016; Smith, Domenech Rodríguez, \& Bernal, 2011). However, a series of difficulties have been reported in cultural adaptation literature.

First, the framework developed by Bernal and colleagues (Bernal et al., 1995; Bernal \& Sáez-Santiago, 2006) has been criticised, particularly because of its list-like format and reported difficulties with implementing the elements in real-world settings (Chu \& Leino, 
2017). The eight elements are not distinct but overlap, e.g. it is hard to differentiate between adaptations made in language or metaphors, which are closely intertwined. In addition, the framework was developed for face-to-face treatments, and its use for other treatment formats such as self-help interventions is limited (Harper Shehadeh et al., 2016).

Second, when looking into the original studies included in the above cited metaanalyses, it becomes evident that such studies tested a large variety of interventions such as psychoeducation, parenting programs, cognitive-behavioural therapy, interpersonal therapy, skills training, systemic therapy, problem solving, etc. The assumed mechanisms of action behind these approaches vary greatly, thus most likely not all of these interventions require the same level of cultural adaptation. What is more, most original studies and meta-analyses do not provide detailed descriptions of the cultural adaptations that were done in the original studies, with some exceptions (e.g., Abi Ramia et al., 2018).

Third, there is very little evidence to determine which cultural adaptation elements are particularly relevant for enhancing treatment acceptability and effectiveness. Benish, Quintana, and Wampold (2011) showed that cultural adaptation of the illness myth, i.e. the explanatory model provided to patients for their symptoms (Bhui, Rudell, \& Priebe, 2006), was the sole moderator of larger effect sizes of culturally adapted psychotherapy when compared to other active treatments $(d=0.21)$. But this finding was based on weak empirical evidence.

Aside from the Bernal framework, a series of other frameworks have been published in the past decade (Domenech Rodríguez \& Bernal, 2012). In an attempt to organize the variety of elements suggested in these frameworks, Chu and Leino (2017) conducted a systematic review and developed a new, data-driven cultural adaptation framework, in which they basically make a distinction between the adaptation of core vs. peripheral aspects in psychotherapy. Core components are the therapeutic ingredients that are assumed to cause symptom change, based on psychological theories, whereas peripheral components include the treatment aspects that are related to the feasibility and acceptability of the intervention (e.g., language or case examples). In their review, Chu and Leino (2017) found that all included studies had implemented peripheral adaptations, whereas $11 \%$ had modified and $60 \%$ had added core components.

The new adaptation framework by Chu and Leino (2017) is an improvement when compared to other frameworks, particularly due to the fact that it was based on original studies rather than expert opinions. Moreover, the division of treatment aspects into peripheral (i.e., engagement and methods of delivery) and core aspects provides an intriguing simplicity in comparison with other frameworks. On the other hand, this framework is based on what has been done so far and therefore cannot capture aspects that have potentially been neglected in literature. Moreover, due to its heuristic nature, it does not provide the necessary theoretical assumptions of how and why cultural adaptation might 
increase the acceptance and effectiveness of psychological interventions. A more theorybased framework can set the ground for empirical research to examine these questions.

When adopting such a theory-driven rather than heuristic perspective, the division between core and peripheral aspects of psychological interventions might not be as straightforward as suggested by Chu and Leino (2017). Two recent prominent systematic reviews conclude that current evidence is insufficient to explain change mechanisms in psychotherapy (Cuijpers, Cristea, et al., 2019; Lemmens, Muller, Arntz, \& Huibers, 2016). It might well be that psychotherapy works through common factors, such as the therapeutic alliance, positive expectations, and a convincing treatment rationale rather than the specific techniques that are assumed to cause changes in symptoms (Cuijpers, Reijnders, \& Huibers, 2019). Thus, factors classified as peripheral by Chu and Leino (2017), e.g. psychoeducation, might actually be the core ingredients of psychotherapy, as is explained more in detail below.

In a more general manner, Resnicow, Baranowski, Ahluwalia, and Braithwaite (1999) differentiate between surface and deep structure adaptations to health interventions. Surface adaptations refer to matching materials (e.g., illustrations, language), as well as channels and settings for treatment delivery to observable characteristics of the target population. By contrast, deep structure adaptations take into account how cultural, social, environmental or historical factors influence health behaviours. Such adaptations are based on assumptions of how members of a particular cultural group perceive the cause, course, and treatment of a particular illness. In other words, and as highlighted by the authors, deep structure conveys salience. Resnicow et al. (1999) developed their framework for health interventions in general. When applying this logic to the cultural adaptation of psychological interventions for the treatment of common mental disorders, deep structure adaptations may take into account results from ethnopsychological studies.

\section{Theoretical and Empirical Foundations for Cultural Adaptation}

Ethnopsychology uses ethnological research to examine different populations' notions of psychological concepts such as the self, emotions, and human nature (White, 1992). Ethnopsychological studies have brought forward a large body of evidence on cultural concepts of distress (CCD), a term that was introduced in DSM-5 to describe local mental health-related phenomena (American Psychiatric Association, 2013). CCD encompass other terms used in literature, such as culture-bound syndromes (American Psychiatric Association, 1994), idioms of distress (Nichter, 1981, 2010), explanatory models (Bhui \& Bhugra, 2002), or illness narratives (Groleau, Young, \& Kirmayer, 2006).

Kohrt et al. (2014) summarised evidence on CCD from different parts of the world in a systematic review. They found that more rigorous studies revealed CCD that clearly dif- 
fered from Western diagnoses of common mental disorders. Such studies examine people's ways of expressing suffering, their assumptions about causes of distress and possible ways to overcome it, physiological and spiritual meanings attributed to suffering, and the distinction between universal human suffering and mental illness (e.g., Keys, Kaiser, Kohrt, Khoury, \& Brewster, 2012; Kohrt \& Hruschka, 2010; Shala, Morina, Salis Gross, Maercker, \& Heim, 2019).

One example of adapting psychological interventions to such CCD was delivered by Hinton, Rivera, Hofmann, Barlow, and Otto (2012), who developed Culturally Adapted Cognitive Behavioural Therapy (CA-CBT) for PTSD. CA-CBT was first developed for Cambodian survivors of the Khmer Rouge. It targets the CCD of khyâl attacks that is based on Cambodians' assumptions about a wind-like substance that circulates in the body (Hinton, Pich, Marques, Nickerson, \& Pollack, 2010). According to this assumption, an imbalance in the khyâl flow causes symptoms such as dizziness and anxiety, which are accompanied by catastrophic beliefs and trauma memories. CA-CBT is based on this particular mind-body concept, and the main treatment components are emotion exposure and emotion regulation techniques (i.e., meditation and yoga-like stretching). Thus, CACBT uses techniques that are not unique for Cambodians, but the treatment rationale provided to patients is rooted in their own explanatory model that is based on khyâl.

This example illustrates one of the basic debates in psychotherapy research, namely the question whether the effect of the treatment is rooted in the techniques themselves, or rather the rationale provided for their use (Wampold \& Imel, 2015). As brought to the point by Wampold (2007) "[p]sychotherapy is not simply the vehicle for the delivery of psychological ingredients but is, rather, a highly entwined system that uses language to construct, or better said, reconstruct the client's interpretations of the world" (p. 8). In psychotherapy research, older and more recent meta-analyses come to the consistent conclusion that after decades of randomised controlled trials (RCTs), we do not know what the "key ingredients" of psychotherapy are (Ahn \& Wampold, 2001; Cuijpers, Cristea, et al., 2019; Lemmens et al., 2016). "Key ingredients" are the treatment components that (are assumed to) cause the symptom change. The current state of the evidence does not allow to conclude whether symptom improvement is caused by specific interventions (e.g., behavioural activation or stress management techniques) or by unspecific factors such as the therapeutic alliance, positive outcome expectations, or providing a convincing treatment rationale (Cuijpers, Cristea, et al., 2019).

This conclusion is highly relevant for research on cultural adaptation of psychological interventions. Chu and Leino (2017) considered "psychoeducation" to be a peripheral aspect of cultural adaptation. However, explaining the purpose of a specific therapeutic technique in a particular way to make it more congruent with the patient's worldview might be much more than just a peripheral adaptation to make the intervention more acceptable. Such adaptations in language might touch on patients' implicit explanatory models, which in turn might change the underlying mechanisms of action, even if the 
intervention itself (e.g., a stress management technique) remains the same. Thus, one and the same adaptation might be considered as core or peripheral.

In summary, theory-driven, experimental studies are needed to better understand whether and how cultural adaptation contributes to the acceptability and effectiveness of psychological interventions. Such studies may in the longer run also contribute to better understand the active ingredients of psychotherapy itself. We aim to lay the groundwork for such studies by suggesting a new conceptual framework. The elements of our framework are based on empirical evidence from ethnopsychological studies, research on the cultural adaptation of psychological interventions, and psychotherapy research outlined above.

\section{A New Framework for Cultural Adaptation}

Our framework is based on the elements of psychological interventions that could potentially be adapted - regardless of whether this has been done in previous research or not. Before conducting empirical studies, it seems important to take a conceptual approach in order to include all aspects of an intervention that might contribute to symptom change.

Our cultural adaptation framework (Figure 1) consists of three main elements which are further described below. We do not formulate pre-assumptions about the components that cause symptom change. Because evidence on substantial modifications is lacking, all elements are considered to be equally relevant for empirical testing. The elements generally reflect the two dimensions suggested by Resnicow et al. (1999), i.e., surface and deep structure adaptations (see above). While Resnicow et al.'s framework was developed more generally for health interventions, we further specified potential deep structure adaptations in psychological interventions for the treatment of common mental disorders.

The elements are presented in what we consider to be a plausible sequence, starting with what may lie at the heart of cultural adaptations, namely the CCD. From CCD - i.e. explanatory models and idioms of distress - relevant treatment components can be derived, and hypotheses can be generated about treatment delivery. In the following, we describe the three main elements and the corresponding sub-elements of the new framework and provide examples from literature to underpin our assumptions. At the same time, we make suggestions on how to implement these adaptations. Our primary aim is to provide a framework as a basis for empirical testing, but the elements outlined below can also be used for adaptations in clinical practice. 


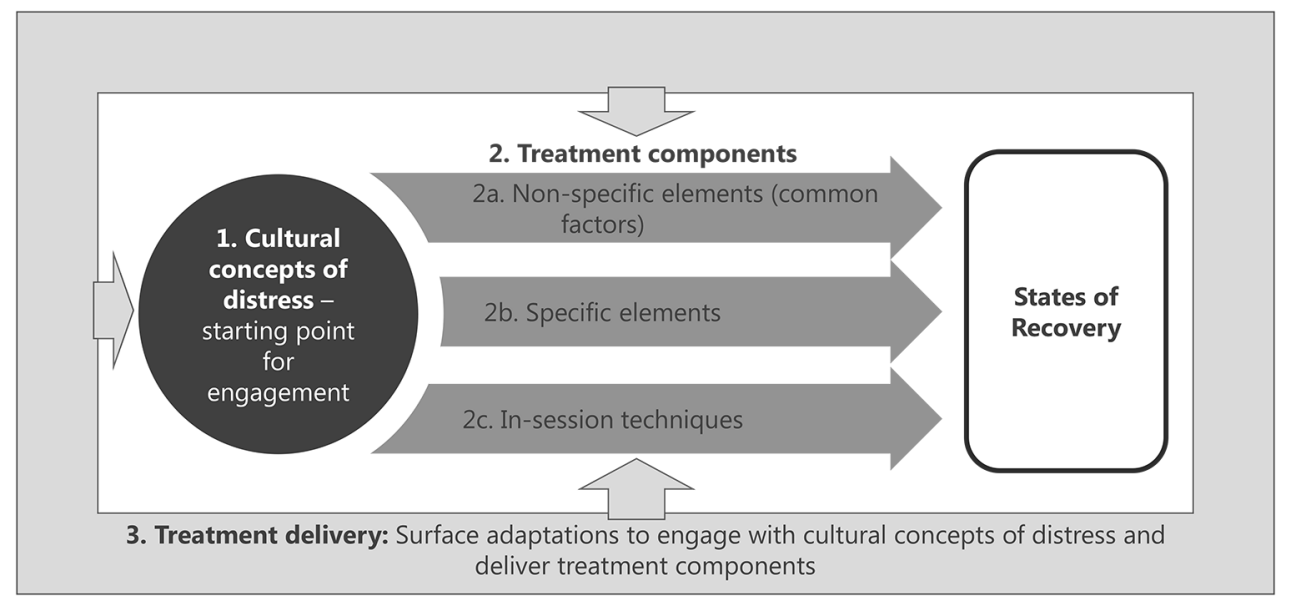

Figure 1. New framework for cultural adaptation.

\section{Cultural Concepts of Distress}

The first element of the framework focuses on core beliefs about human suffering, and the cultural resonance of hypothesized psychological mechanisms of action with ethnotheories of healing. This includes two aspects: Explanatory models (i.e., aetiological assumptions) and idioms of distress (i.e., the expression of symptoms). Several semi-structured interview guidelines have been developed to examine CCD, e.g., the Cultural Formulation Interview in DSM-5 (American Psychiatric Association, 2013), the Short Explanatory Model Interview (SEMI, Lloyd et al., 1998), the Barts Explanatory Model Inventory (BEMI, Rüdell, Bhui, \& Priebe, 2009) or the McGill Illness Narrative Interview (MINI, Groleau et al., 2006). These interviews cover both aspects - idioms of distress and explanatory models - and can help to better understand patients' realities.

a. Explanatory models. People who suffer from psychological distress develop explanations for their symptoms (Wampold, 2007). These explanations are based on intuitive and culturally shaped notions of how mind and body interact (Kirmayer, 2001; Kirmayer \& Bhugra, 2009). Above, we outlined the example of khyâl attacks among Cambodian survivors of the Khmer Rouge (Hinton et al., 2010). Other examples the concept of the heart-mind described in Nepal (Kohrt \& Hruschka, 2010), or the heart narratives related to psychological distress in Haiti (Keys et al., 2012).

Another example are findings related to fatalism. An ethnopsychological study showed that Albanian-speaking immigrants in Switzerland understood their suffering as part of normal life, given by god or fate (fati), and something that cannot be cured but has to be borne with endurance (durim) (Shala et al., 2019). Fatalism was also found among Turkish immigrants in Germany (Franz et al., 2007; Reich, 
Bockel, \& Mewes, 2015). When compared to German patients, Turkish immigrants showed more fatalistic-external control attributions for mental distress, which resulted in lower motivation for psychotherapy. The concept of fate is also described in Islamic understandings of suffering: "The notion of qadar (القَرَر, 'fate') is central to this context. This acceptance of fate should not be equated with fatalism, but can be better understood within a framework of self-abandonment, which is reflected in the value of patience in the face of helplessness and adversity, such as illness and loss. Life may be viewed as a transient phase of existence, a testing place for the eternal life that comes after death" (Hassan et al., 2015, p. 27).

Psychological interventions ideally provide explanations that differ from the patient's own views, but that are not sufficiently discrepant from the patient's intuitive assumptions as to be rejected (Wampold, 2007). For treatment adherence and compliance, it is vital that patients understand and to some point share the rationale behind the treatment. On the other hand, it is also important to provide a new explanation and treatment rationale, in order to motivate patients to try and practice the therapeutic techniques. As an example, Reich, Zürn, and Mewes (2019) developed a web-based intervention to address fatalism and to enhance motivation for psychotherapy among Turkish immigrants in Germany. In a pilot study, they found that this intervention enhanced treatment motivation and reduced fatalistic beliefs.

b. Idioms of distress. This element scrutinises the cultural salience of symptoms that are targeted with an intervention. Common mental disorders are latent (i.e., nonvisible) concepts measured through the expression of symptoms (i.e., their phenomenology). There is a vast body of literature on the difference in symptom expression across cultures, e.g. with regard to emotional vs. somatic complaints (e.g., Kirmayer, 2001; Ma-Kellams, 2014; Ryder et al., 2008). Moreover, ethnopsychological studies from different parts of the world have delivered a broad range of labels used for expressing mental distress in a socially and culturally acceptable manner (e.g., Haroz et al., 2017). As an example, thinking too much is an expression that has been found in many parts of the world and can be used in health communication as a nonstigmatizing way to describe symptoms of psychological distress (Kaiser et al., 2015).

However, it would be erroneous to assume that such labels are simply varying expressions of the same, latent construct (e.g., depression or anxiety). Such local expressions often reflect implicit assumptions about mind-body interactions as described above. Therefore, it is relevant to carefully assess culturally salient symptoms, and to select or target treatment components accordingly.

\section{Treatment Components}

To describe treatment components, we draw on an existing taxonomy developed by Singla et al. (2017), who conducted a systematic review and meta-analysis of psychologi- 
cal interventions in low- and middle-income countries. They applied a multistep analysis of existing taxonomies of common psychological treatment elements and behavioural change techniques used for common mental disorders. Based on this analysis, they proposed a taxonomy of treatment components, which includes the following elements: Specific therapeutic elements (i.e., behavioural, cognitive, interpersonal, and emotional interventions); nonspecific elements to enhance engagement (e.g., empathy, empathic listening, or discussing advantages of and barriers to treatment); and in-session techniques (e.g., goal-setting, role playing, or praising). In their meta-analysis, they found that two specific techniques (i.e., interpersonal and emotional), and nonspecific elements showed the strongest association with trial effectiveness. In the following, we describe how these components may be culturally adapted.

a. Specific elements. Studies testing psychological interventions in low- and middleincome countries have often provided reasons for choosing one technique over another, e.g. arguing that behavioural activation is easier to explain than cognitive techniques, particularly when provided by lay helpers (Dawson et al., 2015). The selection of therapeutic techniques is ideally based on core assumptions about human suffering and healing in the target population, and culturally salient symptoms of psychological distress. As an example, behavioural activation is based on the theoretical assumption that inertia and avoidance are key mechanisms of action in depression (Ferster, 1973; Lewinsohn, 1974; Veale, 2008). However, a qualitative study conducted in Lebanon for the cultural adaptation of an internetbased intervention (Abi Ramia et al., 2018), showed that inertia and inactivity were not key symptoms of depression. Depressed people in Lebanon rather maintain their necessary activities, yet, they were described as becoming irritable, tired, sad, frustrated or angry while continuing to function. This appears to be a global phenomenon: a qualitative systematic review of depression around the world demonstrated similar findings with irritability, anger, and pain figuring prominently but, "[t]he majority of study populations did not raise problems with daily functioning as part of their subjective experiences of depression" (Haroz et al., 2017, p. 160).

In resource-scarce settings where people can simply not afford to become inactive, and where cultural values impede social withdrawal, behavioural activation might not be the first-choice psychological intervention for the treatment of depression. In addition, the focus on improving one's mood through engaging in pleasant activities might not necessarily be a convincing treatment rationale in societies where pursuing affectively positive experiences for oneself is not a key cultural value (Schwartz, 2006).

As another example, Tol et al. (2018) argued that for people in humanitarian settings who suffer from a broad range of symptoms related to psychological distress that cannot be easily categorized as a mental disorder (e.g., nonpathological anxiety, 
grief reactions, and demoralization), general stress management techniques might be more relevant than disorder-specific treatments. Stress management techniques that focus on dealing with negative emotions such as anger, sadness, or nervousness, might be more relevant in such contexts (Hinton et al., 2012; Tol et al., 2018).

b. Nonspecific elements (common factors). Singla et al. (2017) describe these as the elements that are either universal to all treatments, or the ones that are used for enhancing treatment engagement, such as active listening or discussing advantages and disadvantages of the treatment. With regard to elements that are universal to all treatments (e.g., active listening), the cultural adaptation may be limited to surface aspects (see below), such as how active listening is expressed verbally or nonverbally.

When it comes to treatment engagement, providing a convincing and culturally congruent explanatory model may be relevant (see above). For discussing advantages and disadvantages of treatment, it may be relevant to consider culturally-specific notions of stigma, and the way how mental health-related stigma threatens the life domains that "matter most" (Yang, Thornicroft, Alvarado, Vega, \& Link, 2014) to members of a specific cultural group (e.g., marriage, employment, social networks). Advantages and disadvantages of treatment may relate to such culture-specific notions of stigma. People affected by mental disorders could fear stigmatisation if they accept a treatment. On the other hand, patients may realise that treatment and symptom reduction can help in reducing mental health-related stigma, particularly when they are re-integrated into employment or other societal domains.

c. In-session techniques. Singla et al. (2017) subsume a broad range of techniques under this element, such as role-playing, goal setting, homework, or behavioural experiments. Formative research (e.g., key informant interviews or focus groups) can be used to better understand whether such techniques are acceptable in a particular target group, or how these techniques can be adapted to be accessible for members of this target group (Ramaiya, Fiorillo, Regmi, Robins, \& Kohrt, 2017).

\section{Treatment Delivery}

Once the treatment components are defined, the delivery format can be selected, or different formats can be used for different target groups (e.g., face-to-face interventions for older participants and mobile applications for youths). For cultural adaptation of these elements, factors such as literacy level, socio-economic status, gender, or assumptions about the patient-therapist relationship may be taken into account.

a. Delivery format. This element describes cultural preferences and acceptability for different treatment modalities. As an example, several trials have tested the groupbased delivery of potentially scalable interventions as opposed to individual treatment sessions (Epping-Jordan et al., 2016; Sangraula et al., 2018; Verdeli et al., 
2003). Furthermore, internet-based interventions are currently propagated as one potential measure to address the worldwide mental health treatment gap, as they can widely be disseminated among difficult-to-reach populations (Schröder, Berger, Westermann, Klein, \& Moritz, 2016). There is an ongoing debate about the necessity of guidance in internet-based or other self-help interventions (Baumeister, Reichler, Munzinger, \& Lin, 2014). It is theoretically possible that the answer to that question is culturally relative - i.e. that for some cultural groups, guidance is more relevant than for others. Empirical evidence is needed to answer this question.

b. Surface. This element comes closest to what Chu and Leino (2017) considered to be peripheral aspects of psychological interventions, and what Resnicow et al. (1999) described as surface adaptations. A large variety of descriptions of such adaptations has been delivered in literature, such as using culturally adapted language and metaphors (Ramaiya et al., 2017), providing culturally relevant illustrations and case examples (Verdeli et al., 2003), or using easy-to-understand texts (Carswell et al., 2018). Evidence on such adaptations has been summarised in systematic reviews (Chowdhary et al., 2014; Harper Shehadeh et al., 2016). However, so far there is no evidence to show to what extent such adaptations are necessary to enhance acceptance and effectiveness of psychological interventions. Of course, there is a moral obligation not to use treatment materials that are potentially offensive or that may hurt religious feelings. And of course, an intervention is more likely to be accepted when patients feel that the contents are congruent with their own living situations, experiences, and cultural values. But so far, there is insufficient empirical evidence to support this assumption.

\section{Outlook: How to Enhance Empirical Evidence on Cultural Adaptation}

With this new framework, we aim to inspire a theory-driven, empirical approach to cultural adaptation of psychological interventions. A systematic review (Hall et al., 2016) provided indications that culturally adapted psychological interventions are indeed more effective than the unadapted versions of the same interventions. However, there is a lack of evidence on the substantial modifications that cause the higher effectiveness of adapted interventions. In most studies, several aspects were adapted at the same time, and cultural adaptation methods are rarely documented in a replicable manner. In order to advance cultural adaptation research, it would be important to formulate theory-driven hypotheses about the components that are assumed to cause the higher acceptance and effectiveness of adapted interventions, and to test these components using experimental designs.

When developing this framework, we mainly had potentially scalable interventions in mind, i.e., modified, low-intensity and highly standardised evidence-based treatments, which are applied in self-help or guided self-help format, or delivered by lay helpers 
(WHO, 2017). Such interventions are condensed versions of what is done in face-to-face treatments. For such interventions, it is of vital importance to discover which components are most relevant for symptom change, and which aspects are nice-to-have. This also applies to cultural adaptation. For future research and implementation, it is crucial to better understand the treatment elements that have to be culturally adapted to make sure the intervention is acceptable and effective.

The main difference between low- and high-intensity interventions lies - as the names suggest - in the intensity of therapist involvement. In high-intensity interventions, trained therapists can (and most probably do) make "on-the-fly" adaptations whenever working individually with patients from culturally diverse groups. In low-intensity and potentially scalable interventions, most of the treatment aspects are standardised, and in unguided self-help, no contact with a therapist or lay helper is provided at all. In view of transparency and economy of treatments and trainings, it seems helpful to identify the potential cultural adaptations that can and should be made in a standardised manner to ensure that a treatment is acceptable and effective.

In contrast to previous frameworks for cultural adaptation (Bernal et al., 1995; Chu \& Leino, 2017), we used cultural concepts of distress (CCD) as the pivotal point for deep structure adaptation (Resnicow et al., 1999). We suggest starting with an assessment of CCD using semi-structured interviews such as the Cultural Formulation Interview in DSM-5 (American Psychiatric Association, 2013) or the Barts Explanatory Model Inventory (BEMI, Rüdell et al., 2009), and to derive all relevant adaptations from results of such formative research. A desk literature review can help to identify studies that have already assessed CCD in the target population, to avoid duplication of work. However, we intentionally formulated our framework in a way that it does not make pre-assumptions about which adaptations are substantial. It might well be that experimental research (see below) will show that adapting psychological interventions to CCD does not make any difference with regard to their acceptability and/or effectiveness. In our view, it is essential to take this step back and to start with a conceptual framework that includes what seems to be most plausible according to current evidence. From such a conceptual framework, hypotheses can be formulated and tested in empirical studies.

Aside from a new framework, novel research approaches are needed to advance the empirical evidence on the cultural adaptation of psychological interventions. Direct comparison of adapted and unadapted versions of the same interventions are still rather the exception (Hall et al., 2016). This is understandable, as such direct comparisons require very large sample sizes, since small effects are to be expected when comparing two similar treatments with small deviations (Cuijpers, Cristea, et al., 2019). Moreover, training therapists to provide two different versions of the same intervention - adapted and unadapted - is a difficult task. Other treatment formats such as internet or mobile-based interventions, self-help books or audio recordings, are promising for such research. Such highly standardised materials, where input from therapists or lay helpers is minimal, can 
be used to show users two different versions of the same intervention, without large investments in training. Innovative research approaches, e.g. factorial experiments (Collins, 2018) can be used, in which several components are manipulated at the same time. Such research designs can contribute to better understand the substantial modifications in cultural adaptation.

Results from cultural adaptation may also potentially contribute to basic psychotherapy research. For a long time, there has been a debate about the specific and nonspecific components of psychotherapy, and a recent meta-analysis came to the following conclusion: "Based on this set of studies, the only conclusion that can be drawn is that we simply don't know if specific components of specific therapies are effective ingredients of these therapies, or whether all effects are caused by universal, nonspecific factors that are common to all therapies" (Cuijpers, Cristea, et al., 2019, p. 12). Cultural adaptation research provides a promising new approach to this question. As an example, if one and the same intervention (e.g., a stress management technique) shows the same effect, regardless of the explanatory model provided to patients, this is an indicator that the specific intervention caused the symptom change. By contrast, if the same intervention shows a higher effect if it is framed in a culturally-shaped manner, this is an indicator that providing a convincing rationale is indeed a "key ingredient" of psychotherapy, as postulated by Ahn and Wampold (2001). Thus, aside from enhancing access to treatments for culturally diverse groups, cultural adaptation research can make an important contribution to psychotherapy research as a whole.

One important challenge refers to the selection of the target population for cultural adaptation. How do we define a "cultural group"? As an example, Bernal et al. (1995) developed their framework in the context of their work with "Latinos/as" in the United States, and Hinton et al. (2010) worked with "Cambodian refugees". A cultural group can be defined in terms of language, country or region, religion, or other socio-demographic characteristics. Migration is another important aspect, as with time, immigrants start adopting cultural values and norms of the host country, which may be relevant for cultural adaptation of psychological interventions. For research purposes, it is most relevant to carefully define the target population and to be transparent about the criteria according to which this population is defined, to make sure results of studies can be interpreted accordingly. In individual therapy, semi-structured interviews can be used to tailor the interventions to the specific characteristics of the patient.

\section{Conclusion}

Considering the millions of people in need of psychological treatments worldwide (The WHO World Mental Health Survey Consortium, 2004; Turrini et al., 2017), the limited resources available for mental health (Patel et al., 2018), the cultural diversity in common mental disorders (Kohrt et al., 2014), and the variety of treatment components that are potentially relevant for adaptation (Bernal et al., 1995; Bernal \& Sáez-Santiago, 2006; Chu 
\& Leino, 2017), it is vital to expand the empirical evidence as a basis for decision-making on how much and where to invest in cultural adaptation of psychological interventions.

The present paper offers a conceptual framework that lays the groundwork for such empirical research. The three elements suggested in this framework are based on empirical evidence from ethnopsychological studies, cultural adaptation research, and psychotherapy research. Innovative research designs are needed to evaluate the relevance of these elements. Using a theory-driven approach and innovative experimental designs, research on cultural adaptation has the potential not only to make psychological treatments more accessible for culturally adverse groups, but also to further advance empirical research on the basic question of the key ingredients and mechanisms of action in psychotherapy.

Funding: EH is supported by the Swiss National Science Foundation (grant 10001C_169780) and the Swiss Foundation for Psychiatry and Psychotherapy. BAK is supported by the US National Institute of Mental Health (grants K01MH104310, R21MH111280). In addition, both authors are part of Indigo. The Indigo Partnership research programme is a part of the Indigo Network; a collaboration of research colleagues in over 30 countries worldwide committed to developing knowledge about mental-illness-related stigma and discrimination, both in terms of their origins and their eradication. It is coordinated by the Centre for Global Mental Health, Institute of Psychiatry, Psychology and Neuroscience at King's College London. This work was supported in part by the Medical Research Council (grant number MR/R023697/1).

Competing Interests: The authors declare no conflicts of interest.

Acknowledgments: The authors have no support to report.

\section{References}

Abi Ramia, J., Harper Shehadeh, M., Kheir, W., Zoghbi, E., Watts, S., Heim, E., \& El Chammay, R. (2018). Community cognitive interviewing to inform local adaptations of an e-mental health intervention in Lebanon. Global Mental Health, 5, Article e39.

https://doi.org/10.1017/gmh.2018.29

Ahn, H.-n., \& Wampold, B. E. (2001). Where oh where are the specific ingredients? A meta-analysis of component studies in counseling and psychotherapy. Fournal of Counseling Psychology, 48(3), 251-257. https://doi.org/10.1037/0022-0167.48.3.251

Alonso, J., Liu, Z., Evans-Lacko, S., Sadikova, E., Sampson, N., Chatterji, S., . . Thornicroft, G. (2018). Treatment gap for anxiety disorders is global: Results of the World Mental Health Surveys in 21 countries. Depression and Anxiety, 35(3), 195-208. https://doi.org/10.1002/da.22711 American Psychiatric Association. (1994). Diagnostic and statistical manual of mental disorders (4th ed.). Washington, DC, USA: American Psychiatric Association. 
American Psychiatric Association. (2013). Diagnostic and statistical manual of mental disorders (5th ed.). Arlington, VA, USA: American Psychiatric Publishing.

Baumeister, H., Reichler, L., Munzinger, M., \& Lin, J. (2014). The impact of guidance on Internetbased mental health interventions - A systematic review. Internet Interventions, 1(4), 205-215. https://doi.org/10.1016/j.invent.2014.08.003

Benish, S. G., Quintana, S., \& Wampold, B. E. (2011). Culturally adapted psychotherapy and the legitimacy of myth: A direct-comparison meta-analysis. fournal of Counseling Psychology, 58(3), 279-289. https://doi.org/10.1037/a0023626

Bernal, G., Bonilla, J., \& Bellido, C. (1995). Ecological validity and cultural sensitivity for outcome research: Issues for the cultural adaptation and development of psychosocial treatments with Hispanics. Journal of Abnormal Child Psychology, 23, 67-82. https://doi.org/10.1007/BF01447045

Bernal, G., Jiménez-Chafey, M. I., \& Domenech Rodríguez, M. M. (2009). Cultural adaptation of treatments: A resource for considering culture in evidence-based practice. Professional Psychology: Research and Practice, 40(4), 361-368. https://doi.org/10.1037/a0016401

Bernal, G., \& Sáez-Santiago, E. (2006). Culturally centered psychosocial interventions. fournal of Community Psychology, 34(2), 121-132. https://doi.org/10.1002/jcop.20096

Bhui, K., \& Bhugra, D. (2002). Explanatory models for mental distress: Implications for clinical practice and research. The British Journal of Psychiatry, 181(1), 6-7.

https://doi.org/10.1192/bjp.181.1.6

Bhui, K., Rudell, K., \& Priebe, S. (2006). Assessing explanatory models for common mental disorders. Fournal of Clinical Psychiatry, 67(6), 964-971. https://doi.org/10.4088/JCP.v67n0614

Bogic, M., Njoku, A., \& Priebe, S. (2015). Long-term mental health of war-refugees: A systematic literature review. BMC International Health Human Rights, 15, Article 29.

https://doi.org/10.1186/s12914-015-0064-9

Carswell, K., Harper-Shehadeh, M., Watts, S., van't Hof, E., Abi Ramia, J., Heim, E., . . van Ommeren, M. (2018). Step-by-Step: A new WHO digital mental health intervention for depression. mHealth, 4(8), Article 34. Retrieved from http://mhealth.amegroups.com/article/view/20772

Chowdhary, N., Jotheeswaran, A. T., Nadkarni, A., Hollon, S. D., King, M., Jordans, M. J., . . Patel, V. (2014). The methods and outcomes of cultural adaptations of psychological treatments for depressive disorders: A systematic review. Psychological Medicine, 44(6), 1131-1146. https://doi.org/10.1017/S0033291713001785

Chu, J., \& Leino, A. (2017). Advancement in the maturing science of cultural adaptations of evidence-based interventions. Journal of Consulting and Clinical Psychology, 85(1), 45-57. https://doi.org/10.1037/ccp0000145

Collins, L. M. (2018). Optimization of behavioral, biobehavioral, and biomedical interventions: The Multiphase Optimization Strategy (MOST). New York, NY, USA: Springer International Publishing. 
Cuijpers, P., Cristea, I. A., Karyotaki, E., Reijnders, M., \& Hollon, S. D. (2019a). Component studies of psychological treatments of adult depression: A systematic review and meta-analysis. Psychotherapy Research, 29(1), 15-29. https://doi.org/10.1080/10503307.2017.1395922

Cuijpers, P., Karyotaki, E., Reijnders, M., Purgato, M., \& Barbui, C. (2018). Psychotherapies for depression in low- and middle-income countries: A meta-analysis. World Psychiatry, 17(1), 90-101. https://doi.org/10.1002/wps.20493

Cuijpers, P., Reijnders, M., \& Huibers, M. J. H. (2019b). The role of common factors in psychotherapy outcomes. Annual Review of Clinical Psychology, 15, 207-231. https://doi.org/10.1146/annurev-clinpsy-050718-095424

Dawson, K. S., Bryant, R. A., Harper, M., Kuowei Tay, A., Rahman, A., Schafer, A., \& van Ommeren, M. (2015). Problem Management Plus (PM+): A WHO transdiagnostic psychological intervention for common mental health problems. World Psychiatry, 14(3), 354-357. https://doi.org/10.1002/wps.20255

Domenech Rodríguez, M. M., \& Bernal, G. (2012). Frameworks, models, and guidelines for cultural adaptation. In Cultural adaptations: Tools for evidence-based practice with diverse populations (pp. 23-44). Washington, DC, USA: American Psychological Association.

Epping-Jordan, J. E., Harris, R., Brown, F. L., Carswell, K., Foley, C., García-Moreno, C., . . van Ommeren, M. (2016). Self-Help Plus (SH+): A new WHO stress management package. World Psychiatry, 15(3), 295-296. https://doi.org/10.1002/wps.20355

Ferster, C. B. (1973). A functional analysis of depression. American Psychologist, 28(10), 857-870. https://doi.org/10.1037/h0035605

Franz, M., Lujić, C., Koch, E., Wüsten, B., Yürük, N., \& Gallhofer, B. (2007). Subjektive Krankheitskonzepte türkischer Migranten mit psychischen Störungen - Besonderheiten im Vergleich zu deutschen Patienten [Subjective illness beliefs of Turkish migrants with mental disorders - Specific characteristics compared to German patients]. Psychiatrische Praxis, 34(7), 332-338. https://doi.org/10.1055/s-2007-971015

Griner, D., \& Smith, T. B. (2006). Culturally adapted mental health intervention: A meta-analytic review. Psychotherapy: Theory, Research, Practice, Training, 43(4), 531-548. https://doi.org/10.1037/0033-3204.43.4.531

Groleau, D., Young, A., \& Kirmayer, L. J. (2006). The McGill Illness Narrative Interview (MINI): An interview schedule to elicit meanings and modes of reasoning related to illness experience. Transcultural Psychiatry, 43(4), 671-691. https://doi.org/10.1177/1363461506070796

Hall, G. C. N., Ibaraki, A. Y., Huang, E. R., Marti, C. N., \& Stice, E. (2016). A meta-analysis of cultural adaptations of psychological interventions. Behavior Therapy, 47(6), 993-1014. https://doi.org/10.1016/j.beth.2016.09.005

Haroz, E. E., Ritchey, M., Bass, J. K., Kohrt, B. A., Augustinavicius, J., Michalopoulos, L., . . Bolton, P. (2017). How is depression experienced around the world? A systematic review of qualitative literature. Social Science \& Medicine, 183, 151-162.

https://doi.org/10.1016/j.socscimed.2016.12.030 
Harper Shehadeh, M., Heim, E., Chowdhary, N., Maercker, A., \& Albanese, E. (2016). Cultural adaptation of minimally guided interventions for common mental disorders: A systematic review and meta-analysis. 7MIR Mental Health, 3(3), Article e44.

https://doi.org/10.2196/mental.5776

Hassan, G., Kirmayer, L. J., Mekki-Berrada, A., Quosh, C., el Chammay, R., Deville-Stoetzel, J. B., . . . Ventevogel, P. (2015). Culture, context and the mental health and psychosocial wellbeing of Syrians: A review for mental health and psychosocial support staff working with Syrians affected by armed conflict. Retrieved from https://www.unhcr.org/55f6b90f9.pdf

Henrich, J., Heine, S. J., \& Norenzayan, A. (2010). The weirdest people in the world? Behavioral and Brain Sciences, 33(2-3), 61-83. https://doi.org/10.1017/S0140525X0999152X

Hinton, D. E., Pich, V., Marques, L., Nickerson, A., \& Pollack, M. H. (2010). Khyâl attacks: A key idiom of distress among traumatized Cambodia refugees. Culture, Medicine, and Psychiatry, 34(2), 244-278. https://doi.org/10.1007/s11013-010-9174-y

Hinton, D. E., Rivera, E. I., Hofmann, S. G., Barlow, D. H., \& Otto, M. W. (2012). Adapting CBT for traumatized refugees and ethnic minority patients: Examples from culturally adapted CBT (CACBT). Transcultural Psychiatry, 49(2), 340-365. https://doi.org/10.1177/1363461512441595

Hussain-Gambles, M., Atkin, K., \& Leese, B. (2004). Why ethnic minority groups are underrepresented in clinical trials: A review of the literature. Health and Social Care in the Community, 12(5), 382-388. https://doi.org/10.1111/j.1365-2524.2004.00507.x

Kaiser, B. N., Haroz, E. E., Kohrt, B. A., Bolton, P. A., Bass, J. K., \& Hinton, D. E. (2015). "Thinking too much": A systematic review of a common idiom of distress. Social Science \& Medicine, 147, 170-183. https://doi.org/10.1016/j.socscimed.2015.10.044

Keys, H. M., Kaiser, B. N., Kohrt, B. A., Khoury, N. M., \& Brewster, A. R. (2012). Idioms of distress, ethnopsychology, and the clinical encounter in Haiti's Central Plateau. Social Science \& Medicine, 75(3), 555-564. https://doi.org/10.1016/j.socscimed.2012.03.040

Kirmayer, L. J. (2001). Cultural variations in the clinical presentation of depression and anxiety: Implications for diagnosis and treatment. Fournal of Clinical Psychiatry, 62(Suppl 13), 22-28.

Kirmayer, L. J., \& Bhugra, D. (2009). Culture and mental illness: Social context and explanatory models. In I. M. Salloum \& J. E. Mezzich (Eds.), Psychiatric diagnosis: Patterns and prospects (pp. 29-37). New York, NY, USA: John Wiley \& Sons.

Kohrt, B. A., \& Hruschka, D. J. (2010). Nepali concepts of psychological trauma: The role of idioms of distress, ethnopsychology, and ethnophysiology in alleviating suffering and preventing stigma. Culture, Medicine and Psychiatry, 34(2), 322-352.

https://doi.org/10.1007/s11013-010-9170-2

Kohrt, B. A., Rasmussen, A., Kaiser, B. N., Haroz, E. E., Maharjan, S. M., Mutamba, B. B., . . Hinton, D. E. (2014). Cultural concepts of distress and psychiatric disorders: Literature review and research recommendations for global mental health epidemiology. International fournal of Epidemiology, 43(2), 365-406. https://doi.org/10.1093/ije/dyt227 
La Roche, M., \& Christopher, M. S. (2008). Culture and empirically supported treatments: On the road to a collision? Culture \& Psychology, 14(3), 333-356.

https://doi.org/10.1177/1354067X08092637

Lemmens, L. H., Muller, V., Arntz, A., \& Huibers, M. J. H. (2016). Mechanisms of change in psychotherapy for depression: An empirical update and evaluation of research aimed at identifying psychological mediators. Clinical Psychology Review, 50, 95-107. https://doi.org/10.1016/j.cpr.2016.09.004

Lewinsohn, P. M. (1974). A behavioral approach to depression. In R. J. Freidman \& M. Katz (Eds.), The psychology of depression: contemporary theory and research (pp. 157-178). Oxford, United Kingdom: Wiley.

Lloyd, K. R., Jacob, K. S., Patel, V., St Louis, L., Bhugra, D., \& Mann, A. H. (1998). The development of the Short Explanatory Model Interview (SEMI) and its use among primary-care attenders with common mental disorders. Psychological Medicine, 28(5), 1231-1237. https://doi.org/10.1017/S0033291798007065

Ma-Kellams, C. (2014). Cross-cultural differences in somatic awareness and interoceptive accuracy: A review of the literature and directions for future research. Frontiers in Psychology, 5, Article 1379. https://doi.org/10.3389/fpsyg.2014.01379

Mental Health Europe. (2017). World Mental Health Day 2017. Migration: Mental health is not the problem, it's the solution. Retrieved from

https://mhe-sme.org/world-mental-health-day-2017-migration-mental-health-not-problemsolution/

Milat, A. J., King, L., Bauman, A. E., \& Redman, S. (2013). The concept of scalability: Increasing the scale and potential adoption of health promotion interventions into policy and practice. Health Promotion International, 28(3), 285-298. https://doi.org/10.1093/heapro/dar097

Nichter, M. (1981). Idioms of distress: Alternatives in the expression of psychosocial distress: A case study from South India. Culture, Medicine, and Psychiatry, 5(4), 379-408. https://doi.org/10.1007/BF00054782

Nichter, M. (2010). Idioms of distress revisited. Culture, Medicine, and Psychiatry, 34(2), 401-416. https://doi.org/10.1007/s11013-010-9179-6

Patel, V., Saxena, S., Lund, C., Thornicroft, G., Baingana, F., Bolton, P., . . Unützer, J. (2018). The Lancet Commission on global mental health and sustainable development. Lancet, 392, 1553-1598. https://doi.org/10.1016/S0140-6736(18)31612-X

Priebe, S., Giacco, D., \& El-Nagib, R. (2016). Public health aspects of mental health among migrants and refugees: A review of the evidence on mental health care for refugees, asylum seekers and irregular migrants in the WHO European region (Health Evidence Network Synthesis Report, No. 47). Retrieved from https://www.ncbi.nlm.nih.gov/books/NBK391045/

Ramaiya, M. K., Fiorillo, D., Regmi, U., Robins, C. J., \& Kohrt, B. A. (2017). A cultural adaptation of Dialectical Behavior Therapy in Nepal. Cognitive and Behavioral Practice, 24(4), 428-444. https://doi.org/10.1016/j.cbpra.2016.12.005 
Reich, H., Bockel, L., \& Mewes, R. (2015). Motivation for psychotherapy and illness beliefs in Turkish immigrant inpatients in Germany: Results of a cultural comparison study. fournal of Racial and Ethnic Health Disparities, 2(1), 112-123. https://doi.org/10.1007/s40615-014-0054-y

Reich, H., Zürn, D., \& Mewes, R. (2019). Engaging Turkish immigrants in psychotherapy: Development and pilot RCT of a culture-tailored, web-based intervention. (Manuscript submitted for publication).

Resnicow, K., Baranowski, T., Ahluwalia, J. S., \& Braithwaite, R. L. (1999). Cultural sensitivity in public health: Defined and demystified. Ethnicity \& Disease, 9(1), 10-21.

Rüdell, K., Bhui, K., \& Priebe, S. (2009). Concept, development and application of a new mixed method assessment of cultural variations in illness perceptions: Barts explanatory model inventory. Journal of Health Psychology, 14(2), 336-347.

https://doi.org/10.1177/1359105308100218

Ryder, A. G., Yang, J., Zhu, X., Yao, S., Yi, J., Heine, S. J., \& Bagby, R. M. (2008). The cultural shaping of depression: Somatic symptoms in China, psychological symptoms in North America? fournal of Abnormal Psychology, 117(2), 300-313. https://doi.org/10.1037/0021-843X.117.2.300

Sangraula, M., Van't Hof, E., Luitel, N. P., Turner, E. L., Marahatta, K., Nakao, J. H., . . Kohrt, B. A. (2018). Protocol for a feasibility study of group-based focused psychosocial support to improve the psychosocial well-being and functioning of adults affected by humanitarian crises in Nepal: Group Problem Management Plus (PM+). Pilot and Feasibility Studies, 4, Article 126. https://doi.org/10.1186/s40814-018-0315-3

Schröder, J., Berger, T., Westermann, S., Klein, J. P., \& Moritz, S. (2016). Internet interventions for depression: New developments. Dialogues in Clinical Neuroscience, 18(2), 203-212.

Schwartz, S. H. (2006). A theory of cultural value orientations: Explication and applications. Comparative Sociology, 5, 137-182. https://doi.org/10.1163/156913306778667357

Shala, M., Morina, N., Salis Gross, C., Maercker, A., \& Heim, E. (2019). A point in the heart: Concepts of emotional distress among Albanian-speaking immigrants in Switzerland. Culture, Medicine, and Psychiatry. Advance online publication.

https://doi.org/10.1007/s11013-019-09638-5

Singla, D. R., Kohrt, B. A., Murray, L. K., Anand, A., Chorpita, B. F., \& Patel, V. (2017). Psychological treatments for the world: Lessons from low- and middle-income countries. Annual Review of Clinical Psychology, 13(1), 149-181. https://doi.org/10.1146/annurev-clinpsy-032816-045217

Smith, T. B., Domenech Rodríguez, M., \& Bernal, G. (2011). Culture. fournal of Clinical Psychology, 67(2), 166-175. https://doi.org/10.1002/jclp.20757

The WHO World Mental Health Survey Consortium. (2004). Prevalence, severity, and unmet need for treatment of mental disorders in the World Health Organization World Mental Health Surveys. FAMA, 291(21), 2581-2590. https://doi.org/10.1001/jama.291.21.2581

Thornicroft, G., Chatterji, S., Evans-Lacko, S., Gruber, M., Sampson, N., Aguilar-Gaxiola, S., . . . Kessler, R. C. (2017). Undertreatment of people with major depressive disorder in 21 countries. The British fournal of Psychiatry, 210(2), 119-124. https://doi.org/10.1192/bjp.bp.116.188078 
Tol, W. A., Augustinavicius, J., Carswell, K., Brown, F. L., Adaku, A., Leku, M. R., . . van Ommeren, M. (2018). Translation, adaptation, and pilot of a guided self-help intervention to reduce psychological distress in South Sudanese refugees in Uganda. Global Mental Health, 5, Article e25. https://doi.org/10.1017/gmh.2018.14

Turrini, G., Purgato, M., Ballette, F., Nosè, M., Ostuzzi, G., \& Barbui, C. (2017). Common mental disorders in asylum seekers and refugees: Umbrella review of prevalence and intervention studies. International fournal of Mental Health Systems, 11(1), Article 51.

https://doi.org/10.1186/s13033-017-0156-0

UN Dispatch. (2018 December). European Union releases facts and figures for migrant and refugees arrivals in 2018. Retrieved from https://www.undispatch.com/european-union-releases-facts-and-figures-for-migrant-andrefugees-arrivals-in-2018/

Veale, D. (2008). Behavioural activation for depression. Advances in Psychiatric Treatment, 14(1), 29-36. https://doi.org/10.1192/apt.bp.107.004051

Verdeli, H., Clougherty, K., Bolton, P., Speelman, L., Lincoln, N., Bass, J., . . Weissman, M. (2003). Adapting group interpersonal psychotherapy for a developing country: Experience in rural Uganda. World Psychiatry, 2, 114-120.

Wampold, B. E. (2007). Psychotherapy: the humanistic (and effective) treatment. American Psychologist, 62(8), 857-873. https://doi.org/10.1037/0003-066X.62.8.857

Wampold, B., \& Imel, Z. (2015). The great psychotherapy debate. New York, NY, USA: Routledge.

Wendler, D., Kington, R., Madans, J., Wye, G. V., Christ-Schmidt, H., Pratt, L. A., . . Emanuel, E. (2005). Are racial and ethnic minorities less willing to participate in health research? PLoS Medicine, 3(2), Article e19. https://doi.org/10.1371/journal.pmed.0030019

White, G. M. (1992). Ethnopsychology. In C. Lutz, G. M. White, \& T. Schwartz (Eds.), New directions in psychological anthropology (pp. 21-46). New York, NY, USA: Cambridge University Press.

WHO. (2017). Scalable psychological interventions for people in communities affected by adversity - A new area of mental health and psychosocial work at WHO. Retrieved from http://www.who.int/mental_health/management/scalable_psychological_interventions/en/

Yang, L. H., Thornicroft, G., Alvarado, R., Vega, E., \& Link, B. G. (2014). Recent advances in crosscultural measurement in psychiatric epidemiology: Utilizing 'what matters most' to identify culture-specific aspects of stigma. International fournal of Epidemiology, 43(2), 494-510. https://doi.org/10.1093/ije/dyu039 


\section{EACLIPT}

Clinical Psychology in Europe (CPE) is the official journal of the European Association of Clinical Psychology and Psychological Treatment (EACLIPT).

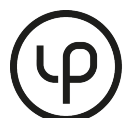

leibniz-psychology.org

PsychOpen GOLD is a publishing service by Leibniz Institute for Psychology Information (ZPID), Germany. 